\title{
Multimonomer Partitioning in Latex Systems with Moderately Water-Soluble Monomers
}

\author{
H. A. S. SCHOONBROOD, ${ }^{1}$ M. A. T. VAN DEN BOOM, ${ }^{1}$ A. L. GERMAN, ${ }^{1 *}$ and J. HUTOVIC ${ }^{2}$ \\ 'Laboratory of Polymer Chemistry, Eindhoven University of Technology, PO Box 513, 5600 MB Eindhoven, \\ The Netherlands, ${ }^{2}$ Sydney University Polymer Centre, School of Chemistry, University of Sydney, \\ Sydney, NSW 2006, Australia
}

\begin{abstract}
SYNOPSIS
Simple equations describing monomer partitioning in latices during intervals 2 and 3 in emulsion polymerization with any number of low to moderately water soluble monomers were derived from the extended Morton equation by making various assumptions. It appears that it is mainly the combinatorial entropy of mixing that governs the partitioning behavior, and that other contributions to the free energy of the monomers in the polymer particles are marginal. Experimental results with styrene, methyl methacrylate, and methyl acrylate confirm the validity of the assumptions. In interval 3 of emulsion polymerization the sum of all contributions to the free energy of the monomers in the particles other than the combinatorial entropy of mixing can be taken as a constant that is dependent only on the monomer composition in the particles and independent of the degree of swelling of the particles. The only parameters one needs to know to calculate the monomer concentrations in all phases with help of the derived equations, are the saturation concentrations of each monomer in the polymer particles, and the saturation concentrations of each monomer in the aqueous phase. (c) $1994 \mathrm{John}$ Wiley \& Sons, Inc.
\end{abstract}

Keywords: multimonomer partitioning • emulsion copolymerization

\section{INTRODUCTION}

The introduction of more than one monomer in emulsion polymerization systems is becoming more and more important in the manufacturing of emulsion polymers. This is simply a result of the recognition that a combination of several monomers in a polymer can result in a wide variety of properties. In quite a lot of cases these properties are superior to the ones obtained by homopolymerizing the single monomers. To make a better polymer by copolymerization one should be able to control the consumption rates of each monomer, i.e., one should be able to control composition drift, as this phenomenon often leads to inferior products. One of the prerequisites for controlling composition drift is knowledge about the thermodynamic partitioning of each monomer between the phases that constitute

* To whom all correspondence should be addressed. Journal of Polymer Science: Part A: Polymer Chemistry, Vol. 32, 2311-2325 (1994) (C) 1994 John Wiley \& Sons, Inc. an emulsion polymerization system (polymer, aqueous, and monomer phases ). It is the thermodynamic partitioning that governs the concentration of each of the monomers in the polymer phase, where most of the polymerization takes place. In this article we present the full equations that describe the partitioning of any number of monomers during intervals 2 and 3 in emulsion polymerization. The equations are based on simplifications to the original Flory-Huggins lattice model and Morton's equation. We will give results of experiments with three monomers that confirm the validity of the derived equations.

\section{THEORY}

In the first section we will summarize the work of other investigators and briefly discuss the more recent work of Maxwell et al. ${ }^{1,2}$ and Noël et al. ${ }^{3}$ who dealt with the partitioning in emulsion systems with one or two monomers. In the second section we will 
derive equations that describe the partitioning of any number of monomers in an emulsion system.

\section{Partitioning with One and Two Monomers}

Several researchers have tried to model monomer partitioning. Morton, Kaizerman, and Altier, ${ }^{4}$ using the Flory-Huggins expression for the free energy of mixing, ${ }^{5-7}$ derived an expression for the saturation swelling of polymer particles by one monomer [eq. (1)]:

$$
\begin{aligned}
\frac{\Delta F_{i p}}{R T}=\ln \left(1-v_{p}\right)+(1- & \left.\frac{1}{M_{n}}\right) v_{p} \\
& +\chi_{i p} v_{p}^{2}+\frac{2 \gamma V_{i}}{r R T}=0
\end{aligned}
$$

$\Delta F_{i p}$ is the partial molar free energy of the monomer in the polymer phase. The first two terms denote the contribution of the combinatorial entropy of mixing (denoted configurational entropy by Flory), the third the heat of mixing and any contributions to the entropy of mixing arising from specific interactions (standard state entropy change). ${ }^{7}$ This term will be referred to as residual free energy of mixing. The fourth term represents the surface free energy. The volume fraction of polymer in the polymer phase is $v_{p}, M_{n}$ is the number-average degree of polymerization of the polymer (usually very large), $\chi$ the Flory-Huggins interaction parameter, $V_{i}$ the molar volume of the monomer, $\gamma$ the interfacial tension between particle phase and aqueous phase, and $r$ is the radius of the particle. This equation will hereafter be referred to as Morton equation, as is customary in literature. Vanzo, Marchessault, and Stannet ${ }^{8}$ took into account the aqueous phase to describe partial swelling, as was later done by Gardon, ${ }^{9}$ setting the left-hand side of eq. (1) equal to $\ln \left(C_{a q} / C_{a q, s}\right)$, the partial molar free energy of the monomer in the aqueous phase $\left(C_{a q}\right.$ is the concentration of monomer in the aqueous phase, and $C_{a q, s}$ is the saturation concentration in the aqueous phase). The result is the so-called Vanzo equation. Ugelstad et al. ${ }^{10}$ extended the Morton equation to be able to describe the monomer partitioning with more than one monomer.

Maxwell et al. ${ }^{1}$ made some assumptions in the Vanzo equation that led to major simplifications [eq. (2)]. The results of these assumptions for the one monomer system are that the partial swelling, especially at higher volume fractions of polymer, can be described by combinatorial entropy of mixing only, i.e., the first two terms on the left-hand side of eq. (1), taking all other terms as a constant correction term [corr. in eq. (2)] that can be calculated from the saturation value of the monomer concentration in the polymer phase. And this parameter is readily accessible from experiment.

$$
\ln \left(1-v_{p}\right)+v_{p}+\text { corr. }=\ln \left[\frac{C_{a q}}{C_{a q, s}}\right]
$$

In a second article Maxwell et al. ${ }^{2}$ worked on the partitioning in a two-monomer system, using the extended equation by Ugelstad et al..$^{10}$ as an expression for the partial molar free energy of monomer $i$ in the polymer phase [eq. (3a) ]. For the partial molar free energy of monomer $i$ in the droplet phase they also used an expression based on the FloryHuggins lattice theory [eq. (3b)] (neglecting the surface free energy term because of the relatively high droplet radii), assuming this can be used to describe the mixing of two low molecular weight components, and for the aqueous phase they applied the right-hand side of the Vanzo equation [eq. (3c)].

$$
\begin{aligned}
\frac{\Delta F_{i p}}{R T}= & \ln \left(v_{i p}\right)+\left(1-m_{i j}\right) v_{j p}+v_{p}+\chi_{i j} v_{j p}^{2}+\chi_{i p} v_{p}^{2} \\
& +v_{j p} v_{p}\left(\chi_{i j}+\chi_{i p}-\chi_{j p} m_{i j}\right)+\frac{2 \gamma V_{i}}{r R T} \\
\frac{\Delta F_{i d}}{R T} & =\ln (3 \mathbf{a})+\left(1-v_{i d}\right) v_{j d}+\chi_{i j} v_{j d}^{2} \\
\frac{\Delta F_{i a q}}{R T} & =\ln \left[\frac{C_{i a q}}{C_{i a q, s, h}}\right]
\end{aligned}
$$

$v_{i p}, v_{i d}$ are the volume fractions of monomer $i$ in the polymer and droplet phase, $m_{i j}$ is the ratio of the molar volumes of monomers $i$ and $j, \chi_{i j}$ is the FloryHuggins interaction parameter between monomers $i$ and $j, \chi_{i p}$ is the interaction parameter between monomer $i$ and polymer, $C_{i a q}$ is the concentration of monomer $i$ in the aqueous phase and $C_{i a q, s, h}$ its saturation value if there are no other monomers present. Maxwell et al. ${ }^{2}$ discussed the difficulties that are inherent with the use of these equations. They made an attempt to simplify these equations in a similar way as they did with the one-monomer system. ${ }^{1}$ Noël et al. ${ }^{3}$ used the same equations and simplifications to derive simple equations for the partial swelling with two monomers. We will briefly go through these simplifications:

1. $m_{i j}$ and its equivalents were set equal to 1 , because the differences in molar volumes for 
many monomers are small. The ratio of the molar volume of monomer $i$ and the molar volume of polymer, $m_{i p}$, and its equivalents were set equal to zero, because the molecular weight of the polymer is much larger than that of the monomers.

2. The contributions of the residual free energy of mixing to the partial molar free energy of mixing two monomers in the monomer or droplet phase, i.e., the third term on the righthand side of eq. ( $3 \mathrm{~b})$, are negligible compared to all other terms.

3 . For the contributions of the residual free energy of mixing to the partial molar free energy of mixing the monomers with the polymer in the particles one can assume the interaction parameters for monomer with polymer to be equal for each monomer. The contribution of the residual free energy of mixing to the mixing of the two monomers in the particles, represented by $\chi_{i j}$, then can be assumed to be negligible, as with the mixing of the monomers by themselves in the droplet phase.

Applying these assumptions to eqs. (3a), (3b) and $(3 \mathrm{c})$ and their equivalents for monomer $j$ and rearranging (for a more detailed treatment see Refs. 2 and 3 ) leads to the following equations, valid under saturation conditions: ${ }^{2,3}$

$$
\begin{aligned}
& \frac{v_{i p}}{v_{j p}}=\frac{v_{i d}}{v_{j d}} \\
& v_{i d}=\frac{C_{i a q}}{C_{i a q, s, h}}
\end{aligned}
$$

Equation ( $4 a$ ) indicates that the saturation value of the concentration of monomer $i$ in the aqueous phase in the presence of monomer $j$ is linearly dependent on the fraction of the monomer in the droplet phase. Since the molar volumes of all monomers are taken to be similar, eq. (4a) leads to eq. (4c). Note that the mole fractions in the polymer phase $\left(f_{i p}\right)$ are related only to the monomers, not to the polymer $\left(f_{i p}=v_{i p} /\left(v_{i p}+v_{j p}\right)\right)$.

$$
f_{i p}=f_{i d}
$$

Because Maxwell et al. ${ }^{2}$ observed a linear dependence of the total monomer concentration in the particles on the monomer mole fraction in the particles, they assumed that the total monomer concentration is equal to the sum of the individual monomer concentrations, and linearly dependent on the mole fractions. This leads to eqs. (4d) and (4e):

$$
\begin{aligned}
C_{p, s} & =\left(C_{i p, s, h}-C_{j p, s, h}\right) f_{i p}+C_{j p, s, h} \\
C_{i p, r} & =f_{i p} C_{p, s}
\end{aligned}
$$

where $C_{i p, s, h}$ is the saturation value of the concentration of monomer $i$ in the polymer phase if only monomer $i$ is present, $C_{i p, r}$ is the concentration of monomer $i$ in the polymer phase in the presence of monomer $j$, and $r$ denotes that the quantity is at its saturation value at the mole ratio $r\left(=v_{i p} / v_{j p}\right.$ or $f_{i p} /$ $f_{j p}$ ) in the polymer or droplet phase. $C_{p, s}$ is the total monomer concentration in the particles.

Similar expressions were derived by Noël et al. ${ }^{3}$ to include partial swelling:

$$
\begin{gathered}
\frac{C_{i a q}}{C_{j a q}}=\frac{C_{i a q, r}}{C_{j a q, r}}=\frac{f_{i p}}{f_{j p}} \frac{C_{i a q, s, h}}{C_{j a q, s, h}} \\
\frac{C_{i p}}{C_{j p}}=\frac{C_{i p, r}}{C_{j p, r}} \\
\ln \left(v_{i p}\right)+v_{p}+\operatorname{corr}_{i}(r)=\ln \left[\frac{C_{i a q}}{C_{i a q, s, h}}\right]
\end{gathered}
$$

The correction factor is assumed to depend only on the monomer mole ratio in the particles, whether at saturation or not, i.e., the value of $\operatorname{corr}_{i}$ is independent of $v_{p}$ or degree of unsaturation.

Maxwell et al. and Noël et al. showed in their articles that the resulting equations hold for several monomer systems [ methyl acrylate (MA)-styrene (S), MA-butyl acrylate (BA), BA-S, vinyl acetateMA]. The validity of eq. (4c) for methyl methacrylate (MMA) and $S$ was already established by Nomura et al., ${ }^{11}$ although they did not derive it theoretically. Aerdts et al. ${ }^{12}$ studied the behavior of MMA and S in swelling poly (MMA-co-S), polybutadiene, and heterogeneous polybutadiene-co[poly (MMA-co-S)]. They found that the equations derived by Maxwell et al. were still valid, even though the polymer systems were very heterogeneous, confirming that the type of polymer does not seriously influence the mixing of the monomers.

In an article by Maxwell et al. ${ }^{13}$ a sensitivity analysis was made showing that deviations from the simple and very straightforward correlations as described by eqs. (4a-e) and ( $5 a-c)$ are within experimental error if the simplifications ( $m_{i j}$ equal to one, $\chi$ terms negligible, surface free energy negligible) are pursued, i.e., if the full equations $(3 \mathbf{a}-\mathbf{c})$ are applied. 


\section{Multimonomer Partitioning with Moderately Water-Soluble Monomers}

The foregoing section dealt with the partitioning of one or two monomers. In the following we shall extend the work of Maxwell et al. ${ }^{1,2}$ and Noël et al. ${ }^{3}$ to obtain equations valid for any number of monomers, $n$. In deriving these equations we shall see that the simplifications made by Maxwell et al. to the Morton equation for systems with one monomer and to the extended equations of Ugelstad for systems with two monomers, can be applied equally well to systems with three or more monomers. This follows from the fact that the assumptions, made by Maxwell et al., leading to rather simple equations describing partial and saturation swelling, ${ }^{1-3}$ can be extended to systems with three monomers or more without any further assumptions.

We shall adopt eqs. (6a-c) as the basic equations that are valid for the systems at hand:

$$
\begin{gathered}
\frac{\Delta F_{i p}}{R T}=\ln \left(v_{i p}\right)+v_{p}+\sum_{\substack{j=1 \\
j \neq i}}^{p} \chi_{i j} v_{j p}^{2}+\sum_{\substack{j=1 \\
j \neq i}}^{n-1} \sum_{\substack{k=j+1 \\
k \neq i}}^{n} v_{j p} v_{k p} \\
\times\left(\chi_{i j}+\chi_{i k}-\chi_{j k}\right)+\sum_{\substack{j=1 \\
j \neq i}}^{n} v_{j p} v_{p} \chi_{i j}+\frac{2 \gamma V}{r R T} \\
\frac{\Delta F i d}{R T}=\ln \left(f_{i d}\right) \\
\frac{\Delta F_{i a q}}{R T}=\ln \left[\frac{C_{i a q}}{C_{i a q, s, h}}\right]
\end{gathered}
$$

In these equations we let $i, j$, and $k$ be $1,2, \ldots, n$ $-1, n$. The number of monomers (or more generally low molecular weight compounds) is $n$, while $p$ represents the polymer in the latex particles. Eq. (6a) was derived from the equation developed by Ugelstad et al. ${ }^{10}$ taking into account the following: We assumed that the molar volumes of the monomers are comparable, so that we can set $m_{i j}$ for each pair equal to 1 [assumption (1), see above]. We replaced $V_{i}$ by $V$, the molar volume of a monomer. It is also justified to set $m_{i p}$ for each monomer equal to 0 , since the molecular weights of the polymers are much larger than those of the monomers. Furthermore, the interaction parameters $\chi_{i p}$ with polymer were taken to be similar for all the monomers [assumption (3), see above].
If the interaction parameters for monomer with polymer are the same for each monomer, then it should be equally valid to set the interactions for each pair of monomers, represented by $\chi_{i j}$ etc., equal. In the following all $\chi_{i j}$ etc. are therefore replaced by $\chi$. We denote this by assumption (3a).

Equation (6b): It was recently shown experimentally ${ }^{14}$ that for $0.5<m_{i j}<2$, eq. (6b) (which is very simple in use) can replace eq. ( $3 \mathrm{~b}$ ) (or rather its equivalent for $\mathrm{n}$ monomers). Since we assume that $m_{i j}=1$ in this article, we can use $f_{i d}$ and $v_{i d}$ interchangeably.

Equation (6c): In principle eq. (6c) is a simplification of the following, more general expression:

$$
\frac{\Delta F_{a q}}{R T}=\ln \left[\frac{\beta_{i} C_{i a q}}{\beta_{i}^{0} C_{i a q}^{0}}\right]
$$

where $\beta_{i}$ is defined as the activity coefficient of monomer $i$, valid at $C_{i a q}, \beta_{i}^{0}$ is defined as the activity coefficient at some standard state, and $C_{i a q}^{0}$ is the concentration at that standard state. The standard state can be defined as the saturated state. However, for concentrations of only a few molar or less, this expression reduces to eq. (3c). We will therefore continue to use eq. (6c) rather than $\left(6 c^{\prime}\right)$.

Let us consider the monomers $x$ and $y$, where $x$ and $y$ can be anything between 1 and $n$. We subtract eq. (6a) for monomer $x$ from its equivalent for monomer $y$ :

$$
\begin{aligned}
& \frac{\Delta F_{x p}-\Delta F_{y p}}{R T}=\ln \left[\frac{v_{x p}}{v_{y p}}\right]+\chi\left(v_{y p}^{2}-v_{x p}^{2}\right) \\
& \quad+\chi\left(v_{y p}-v_{x p}\right) \sum_{\substack{k=1 \\
k \neq x, y}}^{n} v_{k p}+\chi v_{p}\left(v_{y p}-v_{x p}\right)
\end{aligned}
$$

and eq. (6b) for monomer $x$ from its equivalent for monomer $y$

$$
\frac{\Delta F_{x d}-\Delta F_{y d}}{R T}=\ln \left(\frac{f_{x d}}{f_{y d}}\right)
$$

and eq. (6c) for monomer $x$ from its equivalent for monomer $y$

$$
\frac{\Delta F_{x a q}-\Delta F_{y a q}}{R T}=\ln \left(\frac{C_{x a q}}{C_{x a q, s, h}} \frac{C_{y a q, s, h}}{C_{y a q}}\right)
$$


Rearranging eq. (7a) leads to the following:

$$
\begin{aligned}
\frac{\Delta F_{x p}-\Delta F_{y p}}{R T}= & \ln \left(\frac{v_{x p}}{v_{y p}}\right)+\chi\left(v_{y p}-v_{x p}\right) \\
& \times\left[v_{x p}+v_{x p}+\sum_{\substack{k=1 \\
k \neq x, y}}^{n} v_{k p}+v_{p}\right] \\
= & \ln \left(\frac{v_{x p}}{v_{y p}}\right)+\chi\left(v_{y p}-v_{x p}\right)
\end{aligned}
$$

This subtraction can be performed with any combination of monomers 1 to $n$. It is important to realize this, since this fact will be used in the following considerations leading to the same type of equations as those derived by Noël et al. Therefore, the considerations in the next section apply to any monomer.

\section{Saturation Swelling with $n$ Monomers}

Under saturated conditions the following equation holds:

$$
\frac{\Delta F_{i p}}{R T}=\frac{\Delta F_{i d}}{R T}=\frac{\Delta F_{i a q}}{R T}
$$

Eqs. (6b) and (6c) with the right-hand equality of eq. (9) give for monomer $x$ :

$$
f_{x d, c}=\frac{C_{x a q, c}}{C_{x a q, s, h}}
$$

In this equation $c$ denotes the saturation value of the pertaining quantity at that particular composition $c$ of the monomer mixture in the particle or droplet phase. Noël et al. used $r$ to denote this ( $r$ stands for ratio in their work), but since we are deriving equations applicable to more than two monomers, it may be simpler to use $c$ instead, $c$ standing for composition. $c$ represents a particular set of mole fractions in particles and/or droplets: $c=\left\{f_{1}, f_{2}\right.$, $\left.\ldots, f_{n}\right\}$. Since eq. (10) was derived from eqs. (6b) and $(6 \mathrm{c})$, this equation must hold for any monomer.

Combining eqs. ( $7 \mathrm{~b})$ and ( 8 ) we find for saturated conditions:

$$
\begin{aligned}
\ln \left[\frac{v_{x p, c}}{v_{y p, c}}\right]+\chi\left(v_{y p, c}-v_{x p, c}\right) & =\ln \left[\frac{f_{x d, c}}{f_{y d, c}}\right] \\
& =\ln \left[\frac{C_{x a q, c}}{C_{x a q, s, h}} \frac{C_{y a q, s, h}}{C_{y a q, c}}\right]
\end{aligned}
$$

As did Maxwell et al. and Noël et al. we can state that the interaction parameter $\chi$ is small relative to the other terms when mixing monomers (assumption 3). Applying this to the left-hand equality of eq. (11) gives:

$$
\frac{v_{x p, c}}{v_{y p, c}}=\frac{f_{x d, c}}{f_{y d, c}}
$$

Because this applies to any pair of monomers, the following holds also, if we take assumption (1) into account:

$$
f_{x p, c}=\left[\frac{v_{x p, c}}{\sum_{i=1}^{n} v_{i p, c}}=\frac{1}{1+\sum_{\substack{i=1 \\ i \neq x}}^{n} \frac{v_{i p, c}}{v_{x p, c}}}=\frac{1}{1+\sum_{\substack{i=1 \\ i \neq x}}^{n} \frac{f_{i d, c}}{f_{x d, c}}}\right]=f_{x d, c}
$$

This is an important result, because it means that the polymer in the particles has no influence on the mixing of the low molecular weight components, simply because only the combinatorial entropy of mixing is important. Equation (13a) applies to any component. The work of Maxwell et al. ${ }^{2}$ and Noël et al..$^{3}$ clearly showed that eq. (13a) is valid for two monomers. Since we made the same assumptions as they did, we expect the equation to hold for systems with more than two monomers, since it was derived from the same type of equations, but referring to any number of monomers. As was pointed out by Noël et al., the sensitivity analysis performed by Maxwell et al., ${ }^{13}$ showed that the equation can be used, even if not all assumptions hold.

If we can assume that the total monomer concentration in the particles is linearly dependent on the fractions of the monomers in the droplets or particles, ${ }^{2}$ we get the following expression for the total monomer concentration, $C_{p, s}$ :

$$
\begin{aligned}
C_{p, s} & =\sum_{i=1}^{n} f_{i p} C_{i p, s, h} \\
C_{x p, c} & =f_{x p, c}=\sum_{i=1}^{n} f_{i p, c} C_{i p, s, h}
\end{aligned}
$$

From eqs. (10) and (13a) we get:

$$
C_{x a q, c}=f_{x p, c} C_{x a q, s, h}
$$

Note that $C_{x p, c}$ is not only dependent on the mole fraction of $x$, but also on the value of the fractions 
of all other components $\left(\left\{f_{1}, \ldots, f_{n}\right\}\right) . C_{x a q, c}$ is only dependent on the mole fraction of $x$, and independent of the other fractions. This reflects the basic difference between the thermodynamical behavior of the aqueous phase and the monomer and polymer phases. The aqueous phase is so dilute that the monomers do not influence each other [which is why eq. $\left(6 c^{\prime}\right)$ reduces to eq. $\left.(6 c)\right]$; in the monomer and polymer phases, the monomer concentrations are so high that they strongly influence each other. The latter can be simply illustrated by the following example. With eqs. (13b-c) it can easily be shown that the addition of monomer $y$ (under saturated conditions ) to the particles that already contain monomer $x$, can increase the concentration of monomer $x$ to a value greater than $C_{x p, s, h}$ if the ratio $C_{x p, s, h} /$ $C_{y p, s, h}$ is smaller than 0.5 , and inequality (13e) holds:

$$
f_{x p, c}>\frac{1}{\frac{C_{y p, s, h}}{C_{x p, s, h}}-1}
$$

This is shown in Figure 1. In the aqueous phase the addition of another monomer always decreases the concentration of the first monomer. Equations (10) and (13a-d) are very simple to use and characterize the saturated system completely.

\section{Partial Swelling with n Monomers}

For partial swelling (interval 3 in emulsion polymerization, when no monomer droplets are present) we have:

$$
\frac{\Delta F_{i p}}{R T}=\frac{\Delta F_{i a q}}{R T}
$$

Combining this with eqs. (7c) and (8) applying assumption (3) we find for monomer $x$ :

$$
\ln \left(\frac{v_{x p}}{v_{y p}}\right)=\ln \left(\frac{C_{x a q}}{C_{x a q, s, h}} \frac{C_{y a q, s, h}}{C_{y a q}}\right)
$$

From this we get:

$$
\frac{v_{x p}}{v_{y p}}=\frac{f_{x p}}{f_{y p}}=\frac{C_{x a q}}{C_{y a q}} \frac{C_{y a q, s, h}}{C_{x a q, s, h}}
$$

With eq. (13d) this leads to:

$$
\frac{C_{x a q}}{C_{x a q, c}}=\frac{C_{y a q}}{C_{y a q, c}}
$$

This means that the concentrations of all monomers in the aqueous phase in interval 3 will be

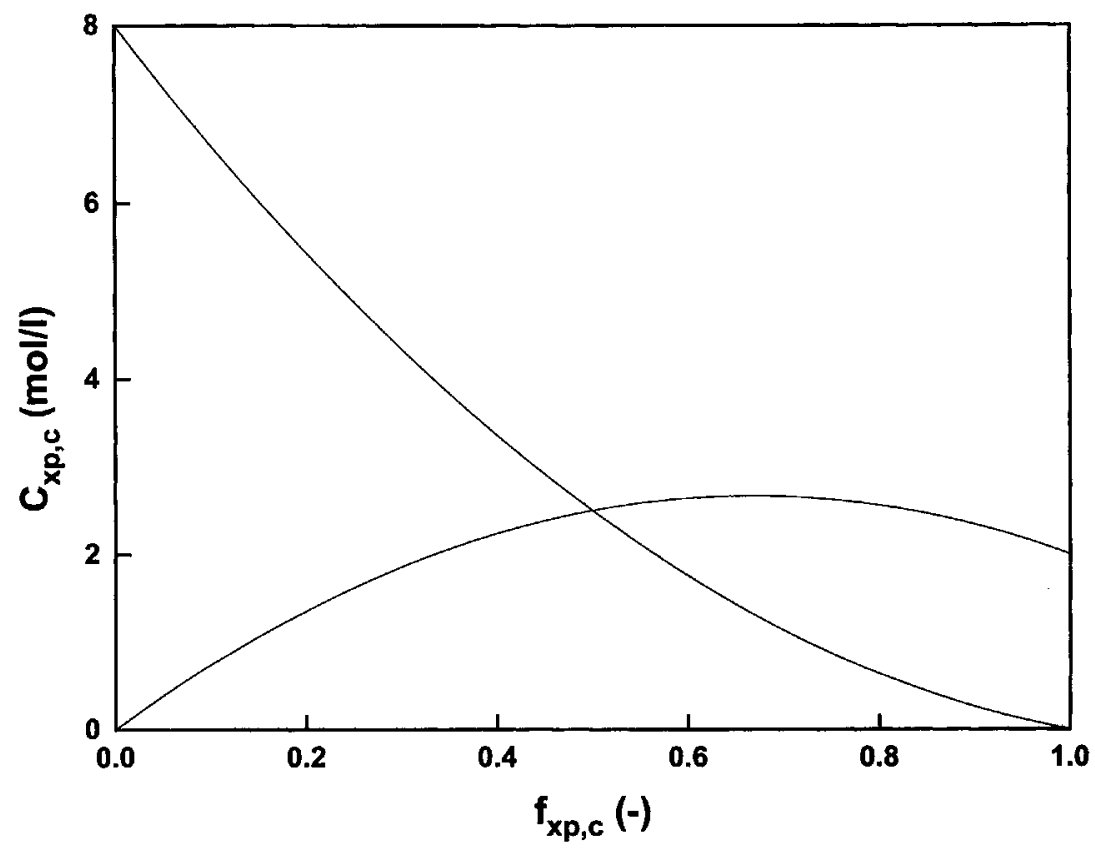

Figure 1. The saturation concentrations in the polymer particles of two monomers for which inequality (13e) holds. $C_{x p, s, h}=2 \mathrm{~mol} / \mathrm{L}, C_{y p, s, h}=8 \mathrm{~mol} / \mathrm{L} . C_{x p, c}>C_{x p, s, h}$ for $f_{x p, c}$ $>1 / 3$. 
equally far from saturation. By definition the following holds as well:

$$
\frac{C_{x p}}{C_{x p, c}}=\frac{C_{y p}}{C_{y p, c}}
$$

$C_{x a q, c}$ and $C_{x p, c}$ are very easily calculated from $C_{x a q, s, h}$ and $C_{x p, s, h}$ if all $f_{x p}$ are given. The above-mentioned equations only give relative concentrations in the polymer and aqueous phases. To obtain the absolute values of the concentrations in the aqueous and polymer phase, one has to go back to eqs. (6a) and (6c) , applying assumption (1), and taking all terms except the combinatorial entropy of mixing together. This results in the next expression:

$$
\ln \left(v_{x p}\right)+v_{p}+\operatorname{corr}_{x}(c)=\ln \left[\frac{C_{x a q}}{C_{x a q, s, h}}\right]
$$

The correction term $\operatorname{corr}_{x}(c)$ represents the terms for the contributions of the surface free energy and the residual free energy of mixing. Maxwell et al. ${ }^{1}$ and Noël et al. ${ }^{3}$ showed that all these terms can be taken constant at every degree of unsaturation, provided that the composition of the monomer mixture (c) does not change, i.e., all $f_{x p}$ stay the same. It is therefore assumed that this term can be calculated from the saturation values of $v_{x p}, v_{p}$ and $C_{x q q}$, at composition $c$ [eq. (17d)]. In turn these can be easily calculated from $C_{x a q, s, h}$ and from $C_{i p, s, h}$ for each monomer $i=1 \ldots n$.

$$
\operatorname{corr}_{x}(c)=-\ln \left(v_{x p, c}\right)-v_{p, c}+\ln \left[\frac{C_{x a q, c}}{C_{x a q, s, h}}\right]
$$

The set of eqs. (17a-d) can be used to characterize the unsaturated case, just as eqs. (13a-d) can be used for the saturated system. The only parameters that need to be known are the values for each $C_{x a q, s, h}$ and $C_{x p, s, h}$.

\section{EXPERIMENTAL}

\section{Chemicals and Seed Latex}

The following monomers were used in our experiments: styrene, methyl acrylate, and methyl methacrylate, all p.a., Merck (Germany). If these monomers were applied in partitioning experiments, they were used as received. Hydroquinone (Merck) was added to prevent polymerization. If polymerizations were carried out with these monomers (i.e., for seed latices ), they were firstly distilled to remove inhibitor by vacuum distillation (and stored at $4^{\circ} \mathrm{C}$ ). The seed latices were prepared with sodium dodecyl sulfate (Fluka, Switzerland) or Aerosol MA80 as emulsifier, sodium bicarbonate (p.a., Merck) as buffer, sodium persulfate (p.a., Fluka) as initiator, and $n$-dodecyl mercaptan (p.a., Merck) as chain transfer agent. The latices that were used were a poly (methyl acrylate) latex [PMA, average diameter $120 \mathrm{~nm}$ (dynamic light scattering)] and a poly (styrene) latex [PS, diameter $86.6 \mathrm{~nm}$ (transmission electron microscopy)].

\section{Monomer Partitioning and Phase Separation Experiments}

For these experiments we mixed a seed latex with a monomer mixture and added extra water. This was then left to stand for $24 \mathrm{~h}$ without agitation, to prevent possible coagulation. Part of this mixture was subsequently transferred to tubes, which were put in an ultracentrifuge. For the experiments with the PMA latex a Centrikon T-2060 was used at 45,000 rpm for 1-2 h. When the PS latex was used the ultracentrifuge was a Sorvall ${ }^{\circledR}$ OTD-65, at 50,000 $\mathrm{rpm}$ for $5 \mathrm{~h}$. The temperature during centrifugation could be controlled. Separation occurred and representative samples of each phase were then analyzed with gas chromatography (GC) Carlo Erba GC 6000 VEGA Series 2 using 2-propanol, iso-butyl methacrylate, and toluene as internal standards or a Hewlett-Packard 5890 with butyl acrylate as an internal standard.

It appeared to be difficult to take representative samples of the aqueous phase in interval 2 (with monomer droplets present). After centrifuging the tubes, the monomer phase was situated on top, the aqueous phase was in the middle part, and the swollen polymer phase was found on the bottom of the tube. (In case of S and PS the polymer phase was situated between the monomer phase and the aqueous phase.) To take a sample of the aqueous phase, a needle had to be inserted through the droplet phase, so care had to be taken to avoid pollution with monomer droplets. A sample of the polymer phase could not be taken without the inclusion of some remains of the aqueous phase. Additionally, it appeared that the swollen polymer phase still contained some "interstitial" aqueous phase, i.e., it was not possible to completely separate the aqueous phase from the particle phase, possibly because the particles retain their round shape during ultracentrifugation. This problem was overcome by drying part of the polymer phase (with included water) to 
determine the solid content. This was used to correct for the amount of water in the sample of polymer phase that was analyzed with GC. However, this means that if there is an equilibrium amount of water present in the particles, this cannot be measured, and it is treated similarly as the water that ends up in the particle phase as a result of the separation method. After determination of the amount of water in the sample, this was excluded from the calculations of monomer concentrations, and by assuming that this amount of water contained the same amounts of monomer as the aqueous phase, we also corrected for an extra amount of monomers in the particles. This amount is usually very small, because of the relatively low concentrations of the monomers in the aqueous phase.
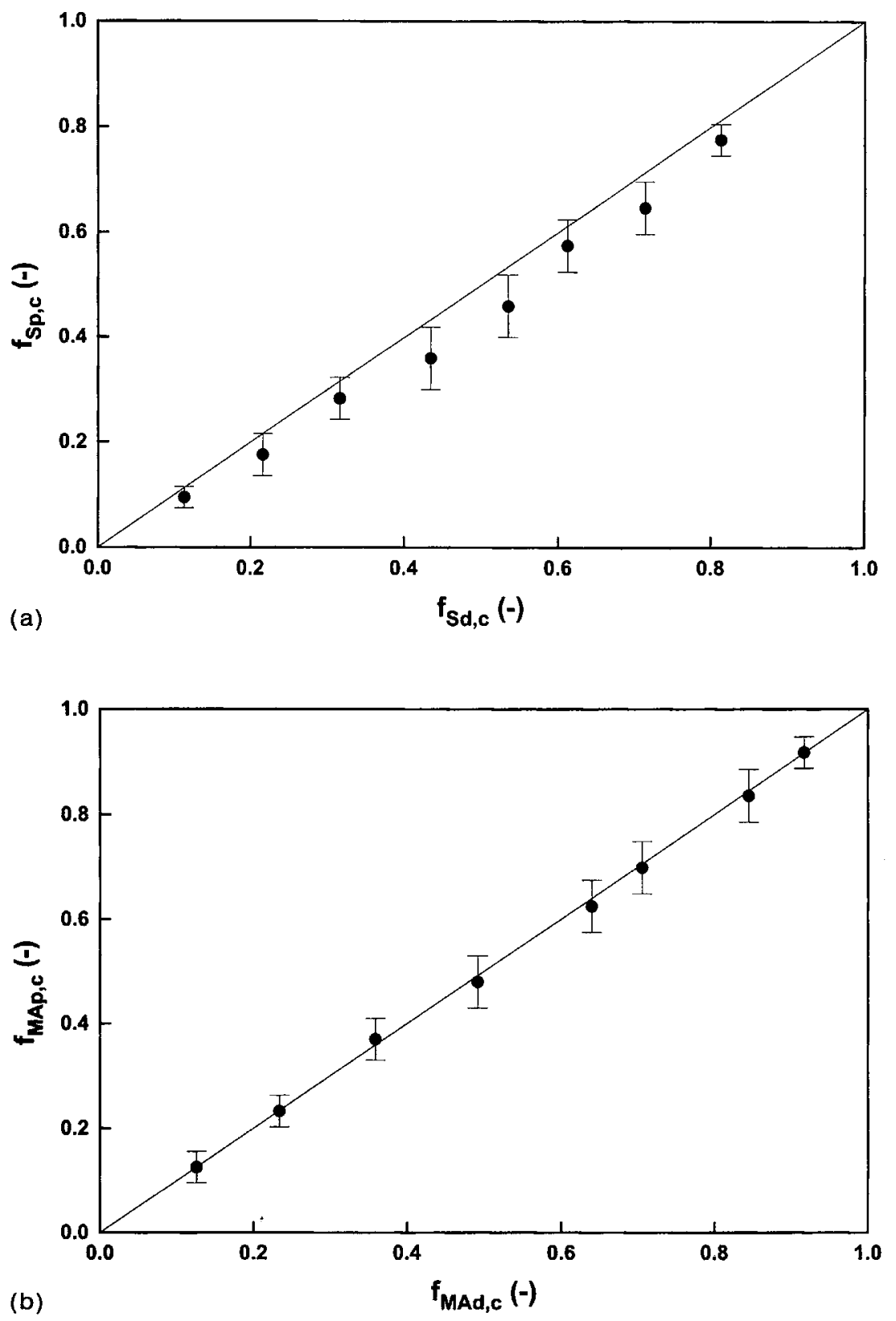

Figure 2. (a) Mole fraction of $S$ in the particle phase $\left(f_{S p, c}\right)$ versus mole fraction of $S$ in the droplet phase $\left(f_{\mathrm{S} d, c}\right)$ in the system S-MMA. The solid line is the prediction by eq. (13a). (b) Mole fraction of MA in the particle phase ( $\left.f_{\text {MAp }, c}\right)$ versus mole fraction of MA in the droplet phase $\left(f_{\text {MAd,c }}\right)$ in the system MA-MMA. The solid line is the prediction by eq. (13a). 


\section{RESULTS AND DISCUSSION}

The monomer system we investigated to check the validity of the various assumptions made in the theory section for the $\mathrm{n}$ monomer systems is styrene (S)-methyl acrylate (MA)-methyl methacrylate (MMA) (these are all low to moderately water-soluble monomers ). All experiments were done at $20^{\circ} \mathrm{C}$.
This section is divided into two parts: swelling with two monomers and swelling with three monomers.

\section{Swelling with Two Monomers}

For swelling with the pairs S-MA and S-MMA of various polymer latices the validity of the assumptions made in this work was already established. ${ }^{1,2,12}$
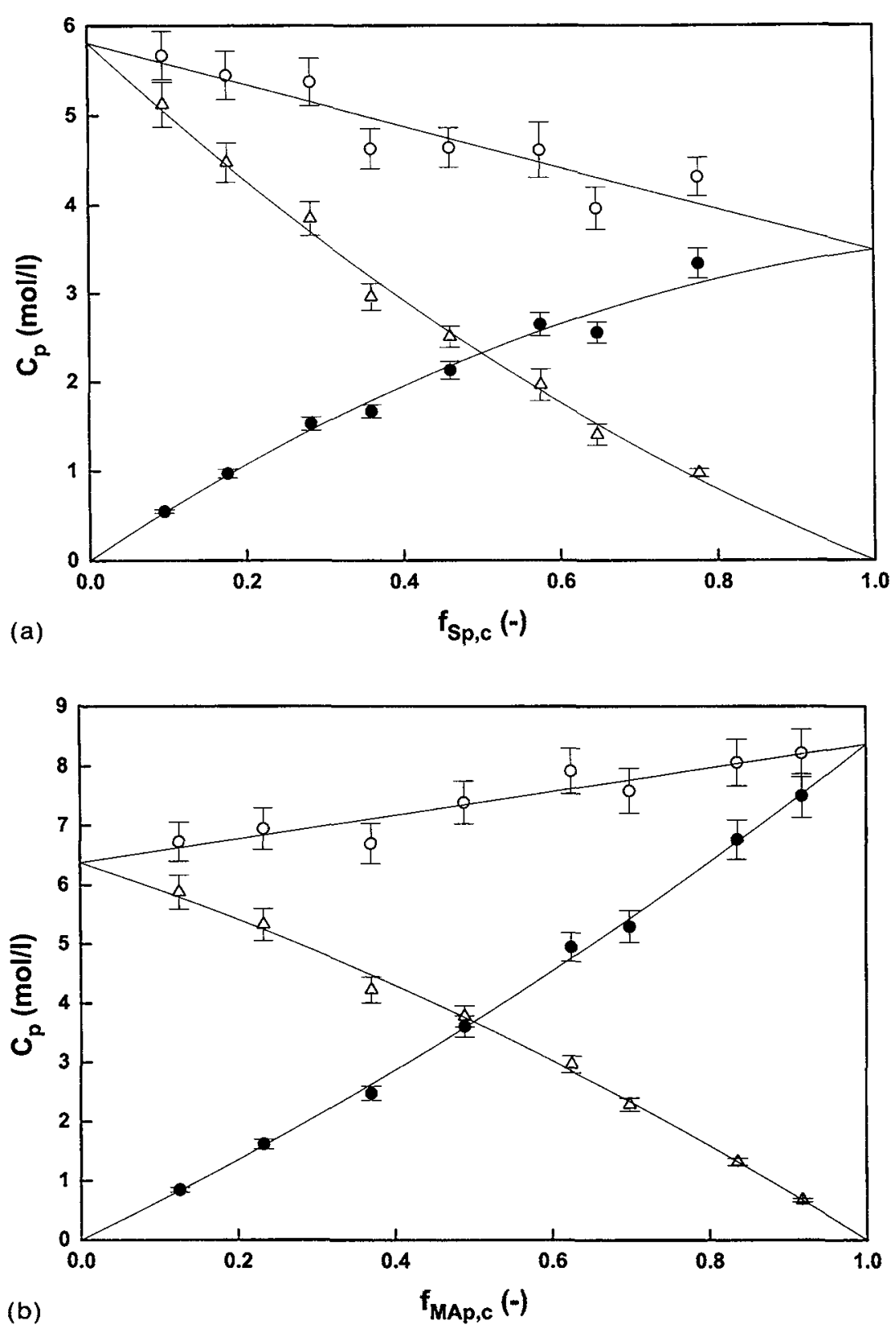

Figure 3. (a) Monomer concentrations in particle phase $\left(C_{p}\right)$ of $\mathrm{S}\left(C_{\mathrm{Sp}, c}\right)(\bullet)$ and MMA $\left(C_{\text {MMAp }, c}\right)(\Delta)$, and total monomer concentration $\left(C_{p, s}\right)(O)$ in the system S-MMA. Solid lines were calculated with eqs. (13b) and (13c), values for $C_{x p, s, h}$ were calculated from these data. (b) Monomer concentrations in particle phase $\left(C_{p}\right)$ of MA $\left(C_{\mathrm{MAp}, c}\right)(\bullet)$ and MMA $\left(C_{\text {MMAp,c }}\right)(\triangle)$, and total monomer concentration $\left(C_{p, s}\right)(O)$ in the system MA-MMA. Solid lines were calculated with eqs. $(13 \mathrm{~b})$ and $(13 \mathrm{c})$, values for $C_{x p, s, h}$ were calculated from these data. 
We checked whether the simplified equations also applied to the S-MMA system and the MA-MMA system on a PMA latex, which were also used in the three monomer swelling experiments. This is shown in Figures 2-4. It can be seen in Figures 2(a) and 2 (b) that also for these systems the mole fractions of monomers in the monomer droplets and polymer particles are the same [eq. (13a)]. The deviation from ideal behavior can be attributed to errors in the gas chromatography. As was noted by Noël et al., ${ }^{3}$ the deviations from ideality that occur when one uses the full equations and reasonable values for all $\chi_{i j}$ are comparable to errors that are usually found for gas chromatography. ${ }^{13}$ This means that
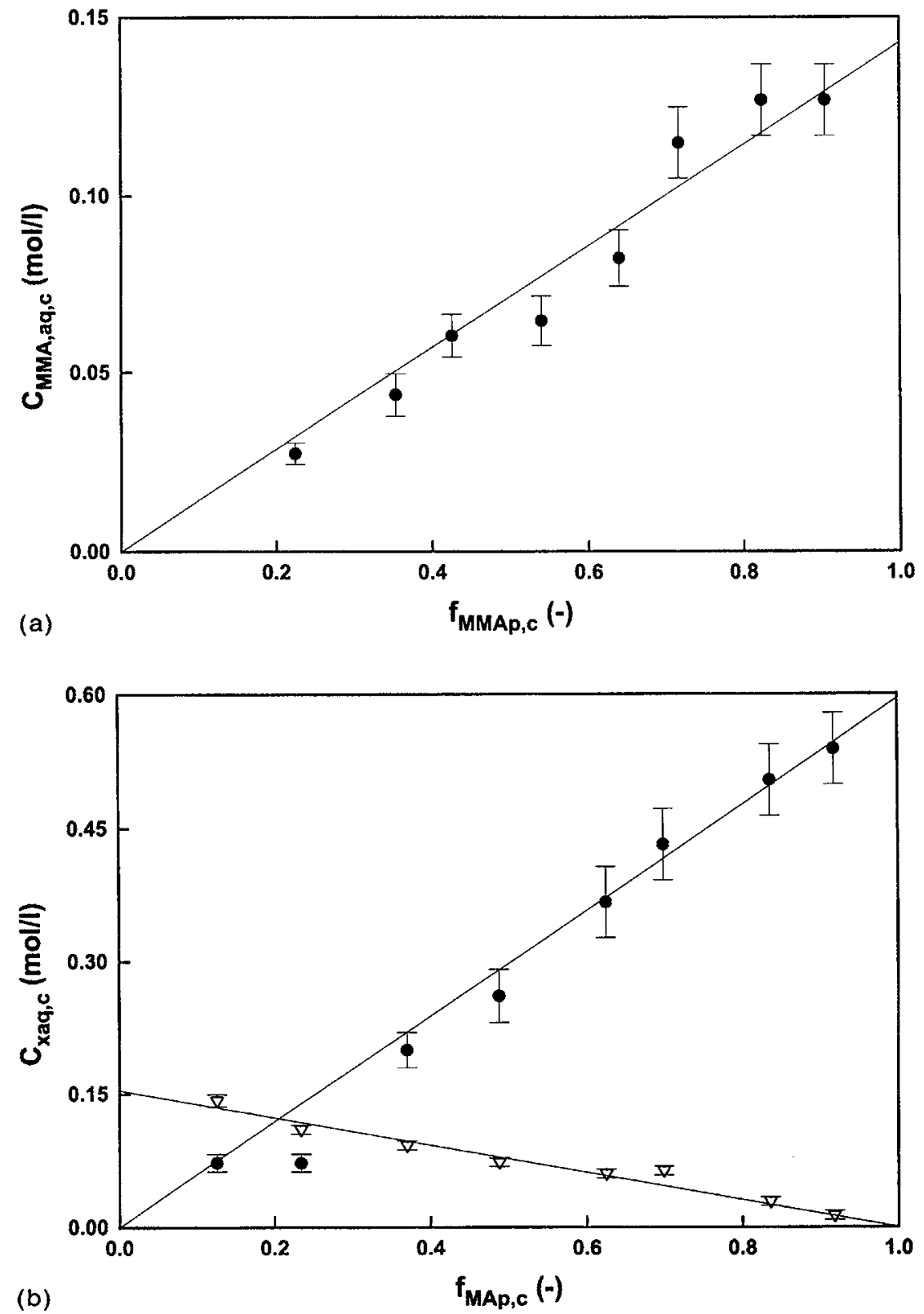

Figure 4. (a) Concentration of MMA in the aqueous phase $\left(C_{\text {MMAaq, }}\right)$ versus mole fraction of MMA in the particle phase ( $f_{\text {MMAp,c }}$ ) (S-MMA system) for which eq. (13d) predicts a

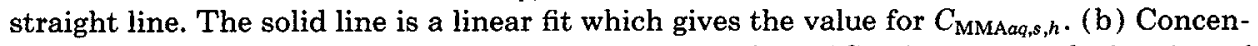
trations of MA (-) and MMA $(\nabla)$ in the aqueous phase $\left(C_{x a q, c}\right)$ versus mole fraction of MA in the particle phase ( $f_{\text {MAp, }}$ ) (MA-MMA system) for which eq. (13d) predicts a straight line. The solid line is a linear fit which gives the values for $C_{\mathrm{MAaq}, s, h}$ and $C_{\mathrm{MMAaq}, s, h}$. 
one cannot distinguish between the simplified equations and the full equations, and that application of eq. (13a) is appropriate even in the case where the assumptions do not hold. Figures $3(a)$ and $3(b)$ show that also eqs. (13b) and (13c) hold. Thus the assumption that the total monomer concentration is a linear function of the mole fraction in the particles is valid. By fitting the data for $C_{p, s}$ with a linear line in Figures 3(a) and 3(b) we found the values for the saturation concentrations of all monomers in the particles of the PMA seed we used (Table I). For comparison we also indicated the values that were found by other investigators: Van Doremaele et al., ${ }^{15}$ Nomura et al., ${ }^{11}$ and Aerdts et al. ${ }^{12}$ Although it is not strictly possible to compare these values, since the polymers in the seeds and the applied temperatures are different and the surface properties will most likely be different as well, it is still possible to discern rough trends. For instance, one could argue that with decreasing polarity of the monomer (going from MA to $\mathrm{S}$ ) the swellability also decreases. However, there is no such trend for the polarity of the seed polymer, i.e., with decreasing polymer polarity (going from PMA to PS) the swellability with a certain monomer does not change regularly. This might be explained by the fact that at saturation the volume fraction of polymer in the particles is lower than the volume fraction of the monomer and therefore the type of monomer has a larger influence than the type of polymer. Typical values for the volume fraction of polymer are: 0.37 for S in PS and 0.24 for MA in PMA. Furthermore, the comparison tells us that the relative low value we found for $S$ in PMA ( $\left.3.49 \mathrm{~mol} / \mathrm{L}, v_{p, s}=0.6\right)$ is not much lower than what was found for BA in PMA or S in PS by Van Doremaele et al. With the saturation concentrations and eq. (13c) we calculated the lines for $C_{x p, c}$. In both figures there is excellent accord with

Table I. Saturation Concentrations of Several Monomers $\left(C_{i p, s, h}\right)(\mathrm{mol} / \mathrm{L})$ in Different Polymer Particles $^{\mathrm{a}}$

\begin{tabular}{lcccc}
\hline Polymer & MA & MMA & BA & S \\
\hline PMA $^{\text {b }}$ & 8.37 & $5.81 / 6.38$ & - & 3.49 \\
PMA $^{15}$ & $7.9 / 8.44$ & - & 3.82 & 5.58 \\
PBA $^{15}$ & 8.75 & - & $5.49 / 6.06$ & 5.36 \\
PS $^{15}$ & 6.22 & - & 5.44 & $4.15 / 6.62$ \\
PS-MMA $^{11}$ & - & 6.9 & - & 5.6 \\
PS-MMA $^{12}$ & - & 6.9 & - & 5.6 \\
\hline
\end{tabular}

Note that the temperatures at which the concentrations were measured varied between 20 and $50^{\circ} \mathrm{C}$.

b This work. the experimental data. The monomer concentrations in the aqueous phase under saturated conditions are linearly dependent on the monomer fractions in the droplets or polymer particles [Figs. 4 (a) and $4(\mathrm{~b})$ ] as predicted by eq. (13d). We ommited the concentrations of $S$ in the aqueous phase, although they were measured. They were found to be very low, as expected from the saturation concentration of $S$ in the aqueous phase, but the reproducibility was not very good because of the low values. The accordance between theory and experiment indicates that the mixing of these monomers with the polymer in the particles is mainly determined by the combinatorial entropy of mixing. It is now firmly established that all assumptions made in this work and in that of Maxwell et al. and Noël et al. are valid the most common monomers in emulsion polymerization.

\section{Swelling with Three Monomers}

\section{Saturation Swelling}

We tested eq. (13a) for the three monomer system S-MMA-MA on the S latex. The results are shown in Figure 5, where we plotted the mole fraction of each monomer in the particle phase versus its mole fraction in the droplet phase, and it can be seen that there is good accordance between experiment and theory, as was the case for the two monomer systems with these monomers. For the same monomer system we measured the saturation concentrations in the aqueous phase as a function of the mole fractions in the particles as a test for eq. (13d). This is shown in Figure 6. We omitted the concentrations of $\mathrm{S}$ for the same reasons as with the two monomer system S-MMA. The average saturation concentrations for MA and MMA in the aqueous phase calculated from extrapolations in Figures 4(a) and 4(b) are 0.584 $\mathrm{mol} / \mathrm{L}$ for MA and $0.157 \mathrm{~mol} / \mathrm{L}$ for MMA, and these values are in good accordance with the values that can be calculated from literature data. ${ }^{16}$ We used these values to predict both lines in Figure 6. For both MMA and MA eq. (13d) holds.

\section{Partial Swelling}

We did several experiments in interval 3 with $S$, MA, and MMA on both the PMA seed and the PS seed. The results are shown in Figures 7 and 8 . In Figure 7 we depicted the ratio of the concentration of MMA in the aqueous phase and its saturation concentration at composition $c$ versus the ratio of 


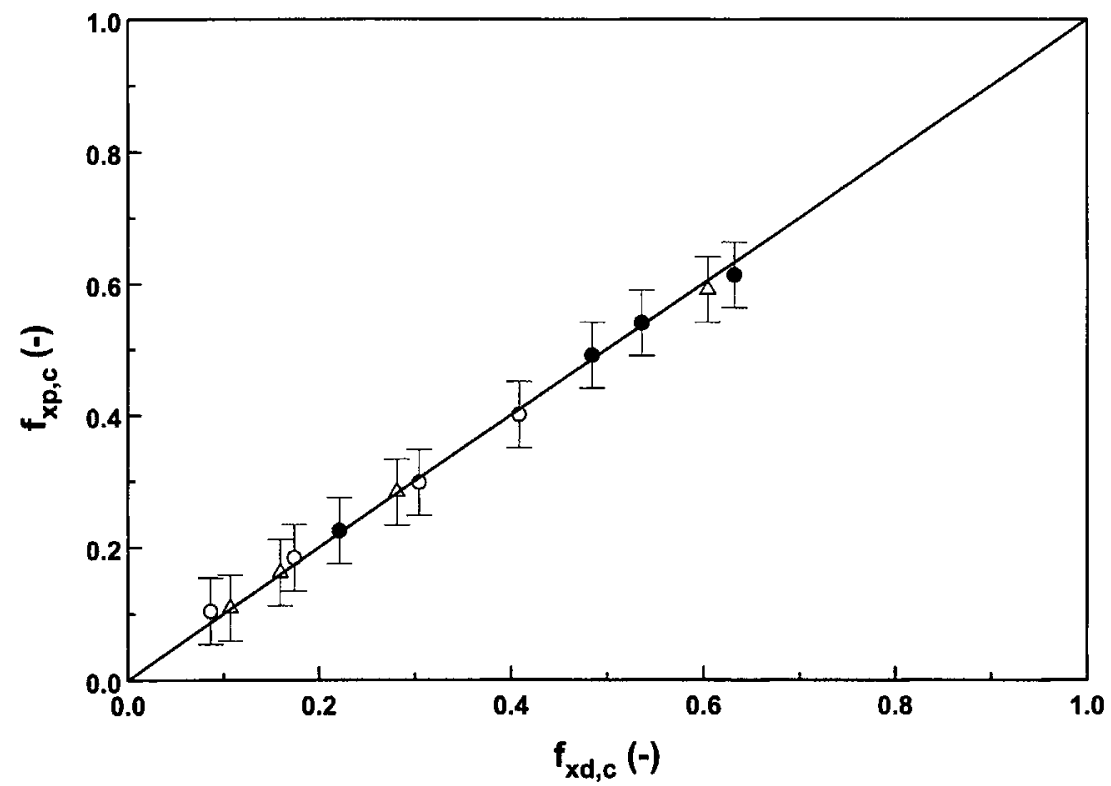

Figure 5. Mole fractions $\left(f_{x p, c}\right)$ of $\mathrm{S}(O)$, MMA $(\triangle)$, and MA $(\bullet)$ in the particle phase versus their respective mole fractions $\left(f_{x d, c}\right)$ in the droplet phase in the system S-MMAMA. The solid line is the prediction by eq. (13a).

the concentration of MA in the aqueous phase and its saturation concentration at the same composition $c$. The composition $c$ in each case was calculated from the concentrations of all three monomers in the particles. According to eq. (17a) this should give a straight line as indicated in the figure. The good accordance indicates that both eqs. (17a) and (16) [ from which eq. (17a) was derived] are valid, which in turn indicates that the assumptions made to derive this equation are valid as well, i.e., partitioning is mainly determined by the combinatorial entropy of mixing.

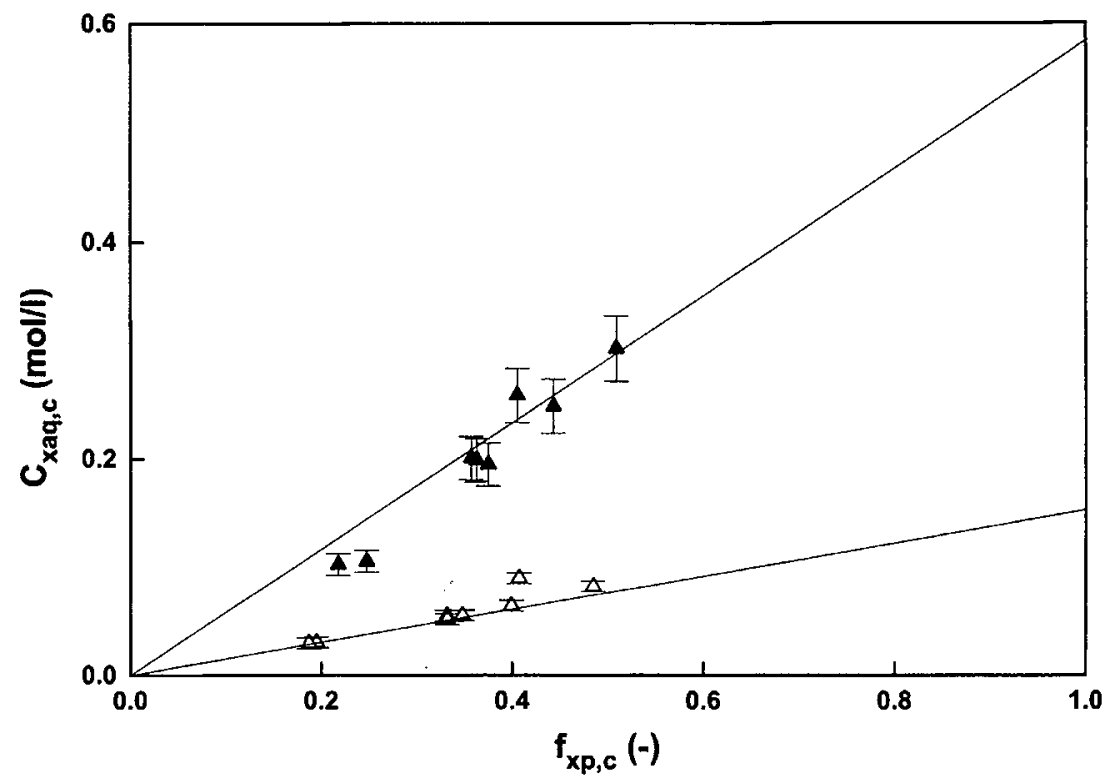

Figure 6. Concentrations of MA $(\Delta)$ and MMA $(\Delta)$ in the aqueous phase $\left(C_{x a q, c}\right)$ versus their respective mole fractions $\left(f_{x p, c}\right)$ in the particle phase for which eq. (13d) predicts a straight line. The solid line is the prediction by eq. (13d). 


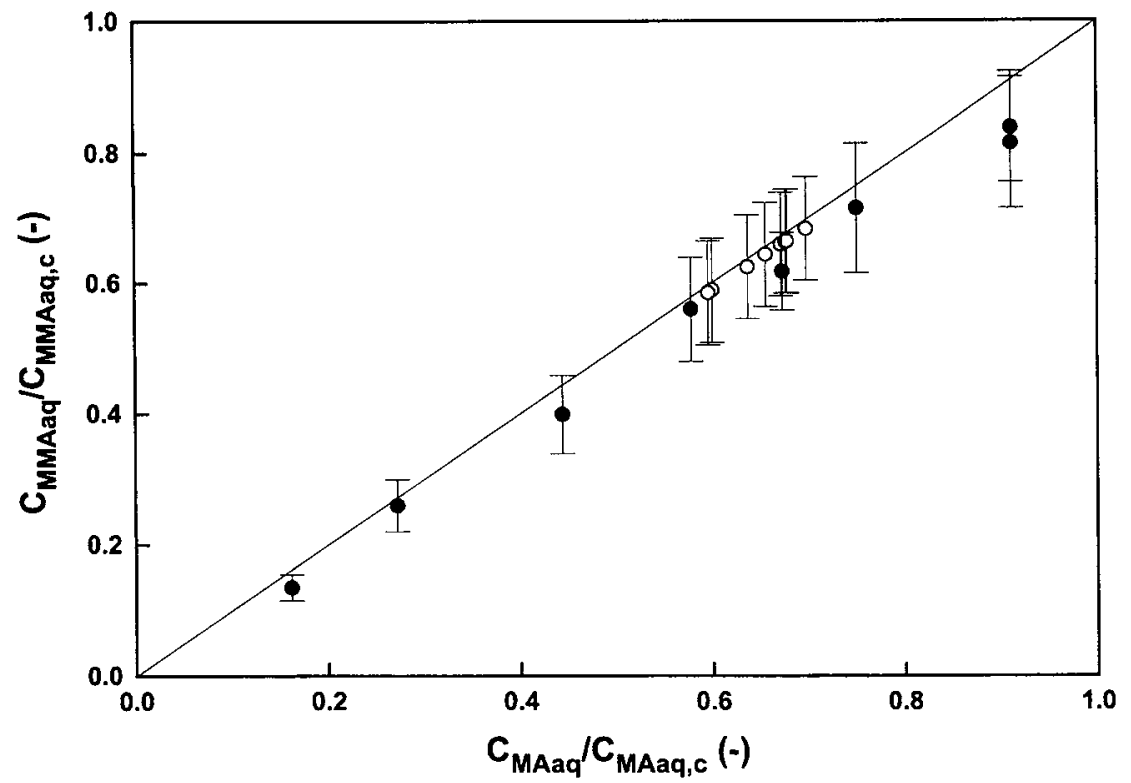

Figure 7. Ratio of the concentration in the aqueous phase of MMA and its saturation value versus the ratio of the concentration of $\mathrm{MA}$ in the aqueous phase and its saturation value: (O) PMA latex, (๑) PS latex. The straight line is the prediction according to eq. (17a).

However, in practice one needs to know the absolute values of the concentrations in both phases, for instance to model emulsion copolymerizations, or to calculate concentrations from mass balances. Equation (16) only gives the ratios of the concen- trations and volume fractions in the aqueous phase and particle phase. Noël et al. ${ }^{3}$ convincingly showed that taking all terms but the combinatorial entropy of mixing (residual free energy of mixing and the contribution of the surface free energy) together as

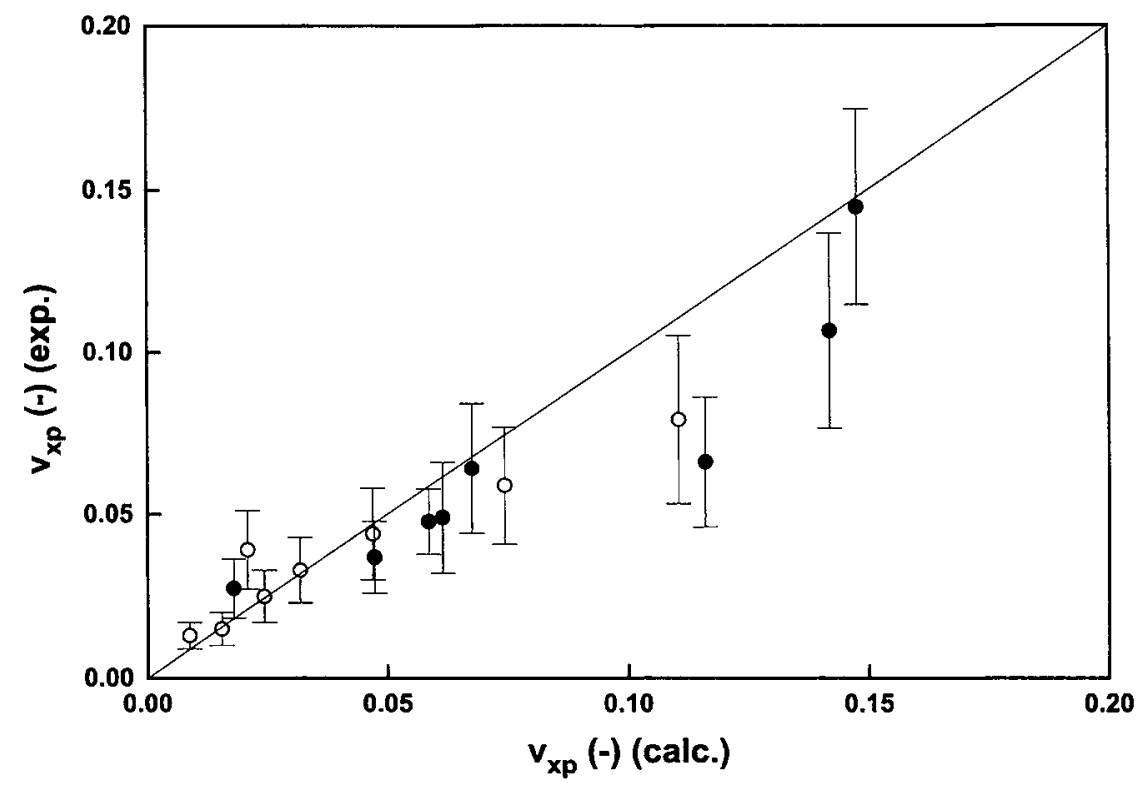

Figure 8. Comparison of calculated values [eqs. (17c) and (17d)] for the volume fractions in the particle phase $\left(v_{x p}\right)$ with measured values for MA $(0)$ and MMA $(\bullet)$. The diagonal line represents exact accordance between calculated and measured values. 
a constant that is dependent only on the composition of the monomer mixture in the particles worked very well for the monomers VAc and MA on a VAc-MAcopolymer seed. In the theory section we derived equations that are based on this concept [eqs. (17c) and $(17 \mathrm{~d})]$.

To check whether these equations can be used in the case of partially swelling a PS seed with S, MMA, and MA we did the following calculations: From experimental data we calculated directly the composition of the monomer mixture $(c)$ in the particles, the volume fraction of polymer in the particles, and the concentrations of the monomers in the aqueous phase. With this information it is possible to calculate the correction terms for both MA and MMA with eq. (17d) ( since the concentrations of $\mathrm{S}$ in the aqueous phase are very low, we did not check it for S). It is then possible to calculate the absolute values of the volume fractions of MMA and MA in the particle phase with eq. (17c). These volume fractions are then compared with the measured values. This comparison is displayed in Figure 8. Because we needed the values for $C_{i p, s, h}$ for each monomer in the PS latex, we determined these in a separate experiment. If eqs. (17c) and (17d) are correct, the measured volume fractions should coincide with the calculated values (and lie on the diagonal). The accordance between calculated and experimental values is quite satisfactory at low volume fractions. Since the calculations are in fact very sensitive to experimental errors (the accuracy of determining the weight fraction of polymer in these experiments was low) the data cannot support nor invalidate the assumptions leading to eqs. (17c) and (17d). Note that this does not invalidate the conclusion that the combinatorial entropy of mixing is the most important contribution to the free energy of mixing.

\section{Restrictions to the Assumptions}

The assumptions made in this and other works ${ }^{1-3,12}$ seem to be valid for monomers with moderate water solubility [including methyl methacrylate (MMA), vinyl acetate (VAc), and methyl acrylate (MA) ${ }^{3}$ ]. However, they have not yet been shown to hold for monomers with a higher water solubility than that of MA (ca. $0.6 \mathrm{M}$ ). Indeed one can expect that the assumption $\chi_{i p}=\chi_{j p}$ no longer holds if one of the monomers has a high polarity, e.g., as is the case with very water-soluble monomers or monomers that are completely miscible in water like 2-hydroxyethyl methacrylate, since the interaction of the monomers with the polymers might not be similar enough for that equality to hold. The same goes for the as- sumption that $\chi_{i j}$ is small. In other words, there might be systems where enthalpy effects and entropy effects arising from specific interactions are not negligible compared with the combinatorial entropy of mixing as opposed to the systems that were checked by Maxwell et al. and Noël et al. It might be reasonable to take the solubility in water as a rough indication of the validity of the assumptions, since the water solubility is inter alia influenced by the polarity of the monomer.

A further assumption is that the chemical potential in the water phase can be described by $\ln \left(a_{i}\right)$ $=\ln \left(C_{i a q} / C_{i a q, s, h}\right)$. If the concentrations of the monomers in the water phase are low, i.e., if the mole fractions are close to 0 , the activity coefficients are close to 1 , and the equation holds. This is equivalent to saying that the monomers have to have a low to moderate water solubility. With higher concentrations, as inevitably occurs with monomers that are miscible in water, deviations start to occur and the equation no longer holds and eq. $\left(6 c^{\prime}\right)$ has to be applied.

Note that the monomers have to have a low to moderate solubility in water, but the equations pertaining to the polymer phase only hold if the monomers are solvents for the polymers as was explicitly stated by Flory. ${ }^{7}$ This is, for instance, not the case with acrylonitrile that is not a solvent for its polymer and therefore does not swell it. However, this means that systems where there is a considerable amount of water imbibed in the particles, cannot be described by these theories, if water is not a solvent for the polymer, which is normally true because it is the actual basis for the existence of polymer latex particles in water. One could argue that in systems where water plays a significant role in the polymer particles, enthalpic interactions are so strong that they cannot be neglected. The assumptions regarding the $\chi$ terms in the theoretical development discussed in the foregoing, are thus not valid. It should, therefore, be realized that in cases where polar, functional monomers are used in high concentrations, the theory probably does not hold.

It is known that water dissolves to a limited extent in both polymers and monomers. ${ }^{16}$ The good accordance that was found between theory and experiment in the articles discussed in the foregoing ${ }^{1-3,12}$ shows that a small amount of water in the particles does not seriously invalidate the theory. In practice, all the water that is found in the particles can be assumed to be part of the aqueous phase, rather than the polymer phase. In conclusion, if water is present only in limited amounts in the particles, it can be shown on both experimental and theoretical grounds 
(Flory ${ }^{7}$ ) that the water should not be treated according to the Flory-Huggins theory, as was done by Tseng et al. ${ }^{16}$ Ugelstad et al. ${ }^{10}$ considered water in the particles in the same way, but noted that the use of the Flory-Huggins equation in this case seems to be open to doubt.

\section{CONCLUSIONS}

The experimental results that are presented show that all assumptions made in this work are valid for the systems investigated experimentally. Monomer partitioning with three monomers in intervals 2 and 3 can be described very well with the simplified equations. It was shown that there are no additional assumptions to be made compared with the two monomer systems. This also indicates that the same equations also hold for systems with more than three monomers. Up until now it has been shown that systems with the monomers most commonly used in emulsion polymerization (styrene, methyl methacrylate, methyl acrylate, butyl acrylate, and vinyl acetate) comply very well to the various conditions set out in the assumptions made in the present and previous articles.

The authors would like to thank Ian Maxwell and Lilian Noël for their useful comments.

\section{REFERENCES AND NOTES}

1. I. A. Maxwell, J. Kurja, G. H. J. Van Doremaele, A. L. German, and B. R. Morrison, Makromol. Chem., 193, 2049 (1992).
2. I. A. Maxwell, J. Kurja, G. H. J. Van Doremaele, and A. L. German, Makromol. Chem., 193, 2065 (1992).

3. L. F. J. Noël, I. A. Maxwell, and A. L. German, Macromolecules, 26, 2911 (1993).

4. M. Morton, S. Kaizerman, and M. W. Altier, J. Colloid Sci., 9, 300 (1954).

5. P. J. Flory, J. Chem. Phys., 10, 51 (1942).

6. M. L. Huggins, J. Phys. Chem., 46, 151 (1942).

7. P. J. Flory, Principles of Polymer Chemistry, Cornell University Press, Ithaca, NY, 1953.

8. E. Vanzo, R. H. Marchessault, and V. Stannet, J. Colloid Sci., 20, 62 (1965).

9. J. L. Gardon, J. Polym. Sci. Polym. Chem. Ed., 6, 2859 (1968).

10. J. Ugelstad, P. C. Mork, H. R. Mfutakamba, E. Soleimany, I. Nordhuus, R. Schmid, A. Berge, T. Ellingsen, O. Aune, and K. Nustad in Science and Technology of Polymer Colloids, G. W. Poehlein, R. H. Ottewil, and J. W. Goodwin, Eds., NATO ASI Series, 1983, Vol. 1, p. 51.

11. M. Nomura, K. Yamamoto, I. Horie, and K. Fujita, J. Appl. Polym. Sci., 27, 2483 (1982).

12. A. M. Aerdts, M. M. W. A. Boei, and A. L. German, Polymer, 34, 574 (1993).

13. I. A. Maxwell, L. F. J. Noël, and H. A. S. Schoonbrood, Makromol. Chem. Theory Simul., 2, 269 (1993).

14. H. A. S. Schoonbrood and A. L. German, Makromol. Chem. Rapid Commun., 15, 259 (1994).

15. G. H. J. Van Doremaele, F. H. J. M. Geerts, H. A. S. Schoonbrood, J. Kurja, and A. L. German, Polymer, 33, 1914 (1992).

16. C. M. Tseng, M. S. El-Aasser, and J. W. Vanderhoff, in Computer Applications in Applied Polymer Science, T. Provder, ACS Symposium Series, Vol. 197, American Chemical Society, Washington D.C. (1982).

Received November 4, 1993

Accepted February 28, 1994 